\title{
IDENTIFICACIÓN DE INULINA Y OLIGOSACÁRIDOS EN LAS HOJAS DE YACÓN (Polymnia sonchifolia) PARA LA OBTENCIÓN DE UN EDULCORANTE*
}

\author{
Cóndor Huamán, Margarita Brígida y Julca Marcelo, Edson Hilmer² \\ Facultad de Ingeniería y Ciencias Humanas. Universidad Nacional del Centro del Perú \\ RESUMEN
}

\begin{abstract}
El objetivo de la investigación fue establecer las condiciones óptimas de extracción de inulina y oligosacáridos en las hojas de yacón, para lo cual se usó la hidrólisis química. Las muestras de hojas de yacón se pesaron y fueron sometidas a cocción a: $80^{\circ} \mathrm{C}, 100^{\circ} \mathrm{C}$ y $120^{\circ} \mathrm{C}$, durante 60,120 y 180 minutos con diferentes soluciones: agua, ácido sulfúrico al $1 \%$ e hidróxido de sodio al $1 \%$. Con este proceso se determinó una casi nula presencia de inulina y de trazas de azúcares que quedaron en el agua de cocción y en las muestras de hojas de yacón homogeneizado por HPLC utilizando un detector de índice de refracción, con columna de intercambio iónico para separación de polisacáridos y ácidos orgánicos transgenomic ICSepICEION-300). El análisis de agua de cocción por HPLC determinó que los niveles de fructosa producidos por hidrólisis de inulina con ácido sulfúrico (1\%) son muy pequeños (sólo 0,025 g/L) al igual que los valores de hidrólisis en agua (hasta 0,25 g/L) e hidróxido de sodio 1\% (hasta 0,13 g/L). En la determinación de niveles de azúcares como xilosa, arabinosa y glucosa se observó que los niveles son similares entre los distintos tratamientos lo que indica que el tratamiento no es muy severo. La concentración de inulina en las muestras homogeneizadas fue menor que en las muestras de agua de cocción.
\end{abstract}

Palabras clave: inulina, oligosacáridos, yacón, hidrólisis

\section{IDENTIFICATION OF INULINI AND OLIGOSACARID IN THE LEAVES OF YACON (Polymnia sonchifolia) FOR OBTAINING A SWEETENER}

\begin{abstract}
The objective of the investigation was to es ytoblish the best conditions inuline and oligosacarid extraction in the YACON leases which were/homogenized by HPLC utilizased in detection the indie de refraction with a column of conic interchange to separate the polisacarids and transgenomic organic acids ICSep ICE-10N-300) elandesis of water for HPLC determined that the of fructose produced by hydrolysis of inuline with sue phuric acid (1\%) are very small (only $0,025 \mathrm{~g} /$ ) the some of the values of an drolysis of water (until $0.25 \mathrm{~g} / \mathrm{l}$ ) sodum hydroxide (1\%), unnl $(0.13 \mathrm{~g} / \mathrm{l})$ in the deterninatin of the levels of sugar with xitosa, arobinosa and glucous, we observed that the levels are similar,amon the different treatments, which means that the treatment is not se the ynuline concentration in thehomopenizes samples, was tess than those in the samples in cooking water.
\end{abstract}

Key word: inuline, oligosacodis, yacón, hydrolipis

\section{INTRODUCCIÓN}

La inulina y la oligofructosa son oligosacáridos derivados de la sacarosa que se aislan de fuentes vegetales como la raíz de la achicoria, la cebolla, el ajo, el puerro o el yacón entre otros; que resultan muy beneficiosos para la salud. Estos ingredientes, además de tener las propiedades clásicas de las fibras alimentarías para regular el tránsito intestinal, contribuyen a la mejor absorción del calcio, a la estimulación de las defensas naturales de la flora intestinal (efecto bífidus) y a reducir el colesterol y los niveles de azúcar en la sangre.

Las principales propiedades de la inulina y oligosacáridos son, en primer lugar, su efecto beneficioso común a la fibra, ya que la ingesta de estos activos a través de los alimentos

\footnotetext{
* Este trabajo de investigación fue recibido el 20/03/2006 retornado para su revisión 20/06/2007 y aprobado para su publicación 10/12/2007

1. E-mail: ideaalesperu@hotmail.com

2. E-mail: hilmer_jm@hotmail.com
} 
contribuye a mejorar la protección y el equilibrio del intestino estimulando la flora intestinal a través de las bifidobacterias. En segundo lugar, mejora la biodisponibilidad del calcio. Esto se traduce en una reducción de la osteoporosis, ya que se ha demostrado que ambos activos vegetales aumentan tanto la densidad mineral del hueso como la masa ósea. $Y$ en tercer lugar, actúan de forma positiva sobre el sistema digestivo ayudando a regular el tránsito intestinal. Por todo esto, la inulina y la oligofructosa son recomendables para todas las personas y especialmente para niños y adolescentes por estar en edad de crecer y de formar su capital cálcico, así como para mujeres en gestación y personas de edad avanzada.

Los datos experimentales muestran que la oligofructosa inhibe la lipogénesis hepática y consecuentemente tiene un efecto hipotrigliceridémico, reduciendo el riesgo de arteroesclerosis. Además, los estudios de estos activos vegetales están demostrando que ayudan a reducir la incidencia de lesiones pre-cancerosas de colon, tanto como el nivel de triglicéridos y de azúcar en sangre. Con lo cual estaría también recomendado su consumo para prevenir diabetes, colesterol y enfermedades cardiovasculares.

El objetivo de la investigación fue establecer las condiciones óptimas de extracción de inulina y oligosacáridos en las hojas de yacón, mediante la hidrólisis química

\section{MATERIAL Y MÉTODOS}

Material y equipos

- Material

a) Materia prima Hojas de yacón amarillo (Polymnia sonchifolia), de la zona de Ulcumayo, provincia de Junín, departamento de Junín.

b) Equipos

- balanza analítica.

- secador de 25 a $60^{\circ} \mathrm{C}$ de 0 a $100 \%$ de humedad Relativa.

- probetas.

- tubos de ensayo.

- pipetas.

- fiolas.

- cápsulas petrie.

- estufa.

- mufla.

- potenciómetro.

- termómetros.

- bioreactor.

- detector de índice de refracción.

- espectrofotómetro.

- texturómetro

\section{- Método de investigación}

Se utilizó dos tipos de investigación: el de tipo correlacional porque se va a manipular y relacionar las diferentes variables (dependientes e independientes) y el de tipo explicativo (estudios experimentales) porque se realizaron las pruebas de laboratorios respectivas tanto para el análisis como para la obtención de inulina.

\section{a) Hidrólisis de inulina}

Este método comprende la hidrólisis del jugo de las hojas de yacón para la separación de la fructosa como complejo cálcico insoluble $\left(\mathrm{C}_{6} \mathrm{H}_{12} \mathrm{O}_{6} \cdot \mathrm{CaO}\right)$, liberación de la fructosa por acidificación del complejo con un ácido que forma una sal cálcica insoluble (ácido carbónico 0 fosfórico), separación de los cationes y aniones contaminantes por medio de resinas de intercambio de iones, concentración en vacío de la solución de fructuosa hasta formar un jarabe espeso y finalmente la cristalización de la inulina.

Sin embargo, para realizar con éxito estas operaciones aparentemente sencillas, deben cumplirse ciertas condiciones de reacción. Las condiciones más importantes son operar a baja temperatura y evitar la excesiva acidez 0 alcalinidad durante todo el proceso. Las muestras de hojas de yacón, se pesaron y fueron sometidas a cocción a: $80^{\circ} \mathrm{C}, 100^{\circ} \mathrm{C}$ y $120^{\circ} \mathrm{C}$, durante 60 , 120 y 180 minutos con diferentes soluciones: agua, ácido sulfúrico al $1 \%$ o hidróxido de sodio al $1 \%$.

La inulina y oligofructosa, no son hidrolizadas por las enzimas digestivas humanas ni absorbidas en la parte superior del tracto gastrointestinal. Su fermentación colónica produce ácidos grasos de cadena corta que podrían ser importantes para mantener la función de las células epiteliales previniendo la carcinogénesis del colon y disminuyen el pH colónico. Al ser ambas fermentadas por los microorganismos del colon estimulan selectivamente las bifidobacterias que inhiben las especies potencialmente patógenas como son los clostridios. Por lo tanto: pueden ser importantes en la resistencia a la colonización y profilaxis de desórdenes gastrointestinales. La inulina posee un sabor neutral suave, moderadamente soluble en agua, otorga cuerpo y palatabilidad. Tiene gran capacidad para reemplazar las grasas. Al mezclarla con agua forma un gel cremoso de excelente palatabilidad.

\section{a) Cromatografía de columna de ligosacáridos}

Pollock (1996) citado por Lizárraga y col. (1997) reporta que la extracción, purificación, caracterización y aplicación de fructooligosacáridos han avanzado 
rápidamente en la última década, debido a las implicaciones en la mejor salud humana y animal. Goto y col., mencionado por Lizárraga y col. (1997) reportan que se han aislado oligosacáridos (desde trisacáridos y dicasacáridos) a partir de raíces de yacón, los cuales fueron purificados por cromatografía de columna y filtración de gel; los cuales serán tomados en cuenta para el presente trabajo.

\section{RESULTADOS}

Con el tratamiento térmico de las hojas de yacón, se determinó una casi nula presencia de inulina y de trazas de azúcares que quedaron en el agua de cocción y en las muestra de hojas de yacón homogeneizado por HPLC utilizando un detector de índice de refracción, con columna de intercambio iónico para separación de polisacáridos y ácidos orgánicos (Transgenomic ICSepICE-ION-300). Se utilizó un estándar de azúcares comerciales de concentración conocida $(1 \mathrm{~g} / \mathrm{L})$ para cuantificar los azúcares presentes (fructosa, glucosa, xilosa y arabinosa). El detector fue de índice. El eluyente utilizado fue una disolución de ácido sulfúrico $0.005 \mathrm{~N}(1.45 \mathrm{ml} / \mathrm{L})$ con un flujo de 0.4 $\mathrm{mL} / \mathrm{min}$; la temperatura de la columna fue de $45^{\circ} \mathrm{C}$ y el volumen de inyección fue de $10 \mathrm{~L}$.

La determinación de furfural se realizó en un espectrofotómetro Shimadzu UV-1601 a 280 nm. Se analizó la textura de las muestras usando un texturómetro (modelo TA XT2i Stable Micro System Texturometer) y la navaja Warner Bratzler con una velocidad de $1 \mathrm{~mm} / \mathrm{seg}$ hasta corte total, para determinar con que tratamiento se podía debilitar los tejidos para la obtención de inulina. Se determinaron los parámetros $L^{*}, a^{*}, b^{\star}, c$ y $\mathrm{H}$ con un colorímetro HunterLab para determinar si ocurrieron cambios en el color debido a los distintos tratamientos y las reacciones de Maillard.

El análisis de agua de cocción por HPLC, determinó que los niveles de fructosa producidos por hidrólisis de inulina con ácido sulfúrico (1\%), son muy pequeños (sólo 0,025 g/L) al igual que los valores de hidrólisis en agua (hasta $0,25 \mathrm{~g} / \mathrm{L}$ ) e hidróxido de sodio $1 \%$ (hasta $0,13 \mathrm{~g} / \mathrm{L}$ ).

En el análisis de las hojas de yacón homogeneizado se observaron mínimos niveles de fructosa en los tratamientos con ácido sulfúrico al $1 \%(0,07 \mathrm{~g} / \mathrm{L})$ y niveles de hasta 0,006 y $0,005 \mathrm{~g} / \mathrm{L}$ en agua e hidróxido de sodio al $1 \%$ respectivamente. Mediante la cuantificación de azúcares ligados a las hojas de yacón después de haber homogeneizado las muestras se observó que los niveles eran todavía más bajos de los vistos en el agua de cocción.

\section{DISCUSIÓN}

Al contrario, de otras plantas, que almacenan los carbohidratos en forma de almidón, el yacón lo hace en forma de inulina u oligofructosa, conocida como alimento de la flora intestinal (función prebiótica), lo cual es favorable para las personas que sufren de diabetes.

Teóricamente, este alimento podría suplantar cultivos como la caña de azúcar y la fructuosa de maíz en muchos productos, desde galletas hasta refrescos, esto con respecto a la raíz.

Otra ventaja se encuentra en sus hojas, que tienen propiedades hipoglicemiantes; pues cuando se bebe un mate de hojas del Yacón se logra reducir la concentración de glucosa en la sangre.

"Un hecho sorprendente - indica Vidal Villagómez Castillo, ingeniero agrónomo del programa de raíces y tuberosas de la Universidad Nacional Agraria La Molina - es que en las zonas donde se consume este producto casi no existen obesos ni diabéticos".

Otros estudios realizados demuestran que las hojas han revelado que entre otros componentes, contiene $11 \%$ de proteína por lo que en los pueblos de la sierra, son utilizadas como forraje para alimentar a animales de pastoreo y para la crianza de cuyes.

En el presente estudio se ha realizado el análisis de las hojas de yacón homogeneizado se observan mínimos niveles de fructosa en los tratamientos con ácido sulfúrico al $1 \%(0,07 \mathrm{~g} / \mathrm{L})$ y niveles de hasta 0,006 y $0,005 \mathrm{~g} / \mathrm{L}$ en agua y hidróxido de sodio al $1 \%$ respectivamente. Mediante la cuantificación de azúcares ligados a las hojas de yacón después de haber homogeneizado las muestras se observó que los niveles eran todavía más bajos de los vistos en el agua de cocción. Los resultados obtenidos indican que no es posible la extracción de inulina y oligosacáridos; y consecuentemente, no es factible la elaboración industrial de un edulcorante con la inulina y oligosácaridos de la hoja de yacón debido a las pequeñas cantidades que se encuentran.

\section{CONCLUSIONES}

- Utilizando como estándar un azúcar comercial con una concentración de $1 \mathrm{~g} / \mathrm{L}$ para cuantificar los azúcares presentes (fructosa, glucosa, xilosa y arabinosa); el detector de índice determinó que los niveles de fructosa producidos por hidrólisis de inulina con ácido sulfúrico (1\%) es sólo 0,025 g/L, que al igual a los valores de hidrólisis en agua es hasta $0,25 \mathrm{~g} / \mathrm{L}$ y en hidróxido de sodio $1 \%$ es hasta $0,13 \mathrm{~g} / \mathrm{L}$.

- En el análisis de las hojas de yacón homogeneizado se observaron en los tratamientos con ácido sulfúrico al 1\% $0,07 \mathrm{~g} / \mathrm{L}$ y niveles de hasta 0,006 y $0,005 \mathrm{~g} / \mathrm{L}$ en agua y en hidróxido de sodio al $1 \%$ respectivamente. 
- Técnicamente no es factible la elaboración de un edulcorante con la inulina y oligosacáridos de las hojas de yacón debido a las pequeñas cantidades que se encuentran en ellas.

- La razón por la cual se le atribuye mayores propiedades para controlar el avance de la diabetes a las hojas de yacón que a las raíces reservantes, es que deben estar brindadas por otros componentes diferentes de la inulina y los oligosacáridos, puesto que el contenido de éstos es mayor en las raíces reservantes que en las hojas debiendo ser mejor la raíz y no las hojas.

\section{LITERATURA CITADA}

Aybar, M., Sánchez Riera, Grau, A. \& Sánchez, S. S. 2001 Hypoglycemic affect of the water extract of Polymnia sonchifolia (yacón) leaves in normal and diabetic rats. Journal of Ethnopharmacology $N^{0} 74$, pp 125-132.

Grau, A. y Rea, J. 1997 Yacón, Smallanthus sonchifoliu (poepp. \& Ende.) H. Robinson. En: Hermann, M. \& Séller J. (Editors). Andean roots and tubers: Ahipa, Arracacha, Maca and Yacón. IPK and IPGRI, Rome, pp 200-242.
León, J. Fundamentos de los cultivos tropicales. Instituto Interamericano de Ciencias Agrícolas (IICA) de la OEA. San José de Costa Rica. pp. 31, 127, 128.

León, J. 1968. Plantas alimenticias andinas. Instituto Interamericano de Ciencias Agrícolas IICA. Boletín Técnico $\mathrm{N}^{0}$ 6. 112p.

Lizárraga, et al. 1997 Centro de investigación en cultivos andinos. Asociación Arariwa. Cusco, Perú.

Ortega, R. et al. 1995 Informe técnico. Subproyecto R2031. Conservación regional de raíces y rizomas en el sur del Perú. Periodo Abril 1994 - Julio Programa colaborativo biodiversidad de RTA's Cusco, Perú.

Programa colaborativo biodiversidad de raíces y tubérculos. 1995. Memorias 1993 - 1994. Consorcio para el desarrollo sostenible de la ecorregión Andina. CONDESAN. La Molina.

Tapia, M. 1990. Cultivos andinos sub - explotados y su aporte a la alimentación. FAO-INIAA. pp. 97-101. 